\title{
A CHARACTERIZATION OF HOMOGENEOUS PLANE CONTINUA THAT ARE CIRCULARLY CHAINABLE ${ }^{1}$
}

\author{
BY C. E. BURGESS
}

Communicated by R. C. Buck, June 18, 1969

It has recently been shown independently, with simultaneous announcements, by Fearnley [10] and Rogers [15] that the pseudoarc $[1],[2],[12],[13]$ is the only nondegenerate homogeneous plane continuum that is both circularly chainable and hereditarily indecomposable. Thus the pseudo-circle, described by Bing [2, p. 48], is not homogeneous. It is the purpose of this note to show that this result by Fearnley and Rogers can be combined with various other theorems to obtain a characterization of all nondegenerate homogeneous plane continua that are circularly chainable. It is interesting that the class of all such continua consists of the three known examples of nondegenerate homogeneous plane continua; namely, the simple closed curve, the pseudo-arc, and the circle of pseudo-arcs [7].

Definitions of linearly chainable continua and of circularly chainable continua can be found in [8]. In some of the references, continua of the former type have been called chainable continua or snake-like continua, and those of the latter type have been called circle-like continua. With the definitions used here, there exist nondegenerate continua which are both linearly chainable and circularly chainable [8, Theorem 7], but in some places in the literature this is not permitted [5, p. 210]. A continuum $M$ is defined to be homogeneous if for each two points $x$ and $y$ of $M$ there is a homeomorphism of $M$ onto itself that takes $x$ onto $y$.

It is our intention to include enough references to indicate a complete proof of the following theorem, even though some parts of the proof may already be known to people who are familiar with the references. A history of work on the problem of classifying homogeneous plane continua can be found in [7].

TheOREM. A nondegenerate circularly chainable plane continuum is homogeneous if and only if it is either a simple closed curve, a pseudoarc, or a circle of pseudo-arcs.

Proof. A pseudo-arc has been characterized by Bing [2] as a linearly chainable continuum that is hereditarily indecomposable. Bing

1 This work was supported by the National Science Foundation under NSF-GP7058. 
[1] and Moise [14] have shown that the pseudo-arc is homogeneous. Also, it is circularly chainable [8, Theorem 7]. Bing and Jones [7] described a circle of pseudo-arcs and proved that it is homogeneous. Each such continuum is circularly chainable, by definition, and any two of them are homeomorphic [7, p. 189]. Thus each of the three types of plane continua mentioned in the theorem is both homogenous and circularly chainable.

Let $M$ be a nondegenerate plane continuum which is both homogeneous and circularly chainable. Two cases will be considered, depending upon whether $M$ is decomposable or indecomposable.

Case 1. $M$ is decomposable. Jones [11, Theorem 2] has shown that every homogeneous nondegenerate decomposable plane continuum is a "circle of homogeneous tree-like continua." Since $M$ is circularly chainable, each of these tree-like continua is linearly chainable and hence must be either a point or a nondegenerate homogeneous continuum that is linearly chainable. Bing [4] has shown that every continuum of the latter type is a pseudo-arc. Thus $M$ is either a simple closed curve or a circle of pseudo-arcs.

Case 2. $M$ is indecomposable. It follows from [9, Theorem 11], together with [6, Theorem 7], that $M$ is hereditarily indecomposable. If $M$ were to separate the plane, it would be a pseudo-circle. Fearnley [10] and Rogers [15] have shown that such a continuum cannot be homogeneous. Thus $M$ does not separate the plane and, consequently, is tree-like [3, Theorem 6]. It follows from [8, Theorem 10] that $M$ is linearly chainable. Now since $M$ is hereditarily indecomposable, it must be a pseudo-arc [2, Theorem 1$]$.

\section{REFERENCES}

1. R. H. Bing, A homogeneous indecomposable plane continuum, Duke Math. J. 15 (1948), 729-742.

2. - Concerning hereditarily indecomposable continua, Pacific J. Math. 1 (1951), 43-51.

3. - Snake-like continua, Duke Math. J. 18 (1951), 653-663.

4. - Each homogeneous nondegenerate chainable continuum is a pseudo-arc, Proc. Amer. Math. Soc. 10 (1959), 345-346.

5. - A simple closed curve is the only homogeneous bounded plane continuum that contains an arc, Canad. J. Math. 12 (1960), 209-230.

6. ㄴ., Embedding circle-like continua in the plane, Canad. J. Math. 14 (1962), 113-128.

7. R. H. Bing and F. B. Jones, Another homogeneous plane continuum, Trans. Amer. Math. Soc. 90 (1959), 171-192.

8. C. E. Burgess, Chainable continua and indecomposability, Pacific J. Math. 9 (1959), 653-659.

9. - Homogeneous continua which are almost chainable, Canad. J. Math. 13 (1961), 519-528. 
10. Lawrence Fearnley, The psuedo-circle is not homogeneous, Bull. Amer. Math. Soc. 75 (1969), 554-558. Notices Amer. Math. Soc. 15 (1968), Abstract \#68T-G25, 942.

11. F. B. Jones, On a certain type of homogeneous plane continuum, Proc. Amer. Math. Soc. 6 (1955), 735-740.

12. B. Knaster, Un continu dont tout sous-continu est indécomposable, Fund. Math. 3 (1922), 247-286.

13. E. E. Moise, $A n$ indecomposable plane continuum which is homeomorphic to each of its nondegernerate subcontinua, Trans. Amer. Math. Soc. 63 (1948), 581-594.

14. - A note on the pseudo-arc, Trans. Amer. Math. Soc. 64 (1949), 57-58.

15. J. T. Rogers, Jr., The pseudo-circle is not homogeneous, Notices Amer. Math. Soc. 15 (1968), 943.

University of Utah, Salt Lake City, Utah 84112 